\title{
Localization-delocalization transition in disordered one-dimensional exciton-polariton system
}

\author{
(C) A.V. Larionov ${ }^{1}$, A.S. Brichkin ${ }^{1}$, S. Höfling ${ }^{2}$, V.D. Kulakovskii ${ }^{1,3,9}$ \\ ${ }^{1}$ Institute of Solid State Physics, Russian Academy of Sciences, \\ 142432 Chernogolovka, Russia \\ ${ }^{2}$ Technische Physik, Universität Würzburg, \\ D-97074Würzburg, Germany \\ ${ }^{3}$ National Research University Higher School of Economics, \\ 101000 Moscow, Russia \\ ` E-mail: kulakovs@issp.ac.ru
}

The transition from the delocalized to the localized state has been investigated in a quasi-onedimensional exciton-polariton system excited nonresonantly in GaAs-based microcavity wire with disordered potential.The photoexcited polariton condensate has been found to spread along the wire with a velocity exceeding $1 \mu \mathrm{m} / \mathrm{ps}$. The propagation along the wire is provided by high energy polaritons. The LP localization length decreases with decreasing blue shift of LPs in the excited spot. The polariton condensate returns to the Bose glass state when the blue shift of the LP resonance at the excitation spot decreases below the critical level that depends on the potential disorder.

\section{Acknowledgements}

We are grateful to S.S. Gavrilov, S.G. Tikhodeev, and V.B. Timofeev for fruitful discussions and C. Schneider for the MC samples. The work was partly supported by the Russian Foundation for Basic Research (Grant No 16-02-00407) and the State of Bavaria. 